\title{
Projeto PerGRam - Processos para o ensino da gramática no primeiro ano de escolaridade
}

\author{
Míriam Pessôa Marques \\ Universidad Federal de Alagoas
}

(Texto recibido 25 de julio de 2019; aceptado 25 de julio de 2019)

DOI: https://doi.org/10.5565/rev/jt13.814

En el Proyecto PerGRam, el equipo formado por Susana Pereira, Adriana Cardoso, Clara Nunes Correia, Patrícia Ferreira, Teresa Leite, Mariana Pinto, Encarnação Silva y Filomena Viegas propone un conjunto de trayectos didácticos con el objetivo de estimular la reflexión y la investigación sobre los procesos que implican la enseñanza y aprendizaje de la gramática en los primeros años de la escuela en Portugal, reconociendo que en el ámbito de la enseñanza de la lengua materna se ha prestado poca atención a la gramática en el aula y teniendo en cuenta las necesidades escolares en el contexto del Portugués como L1 ${ }^{i}$. Para eso, dos cuestiones fueran orientadoras para el desarrollo del proyecto: a) ¿Qué tipo de enseñanza promueve la actividad metalingüística, para evolucionar el nivel de consciencia lingüística?; b) ¿Cuáles las necesidades de formación y qué modelos de formación pueden potenciar las mudanzas de las prácticas en el aula? (Cardoso et al., 2018).

El proyecto tiene como prioridad la creación y comprobación de un abordaje innovador para el desarrollo de las competencias reflexivas sobre la lengua en los ámbitos de enseñanza y aprendizaje de la gramática y formación de profesionales del área. A partir de las cuestiones orientadoras para su elaboración, se plantearon dos objetivos específicos: a) proponer un trayecto didáctico pensado para promover la actividad metalingüística en el primer año de la escuela, que resultó en la elaboración de recursos didácticos susceptibles de experimentación y basados en investigaciones actuales de didáctica de la enseñanza de la gramática en L1; b) explorar a partir de ello nuevas metodologías para promover el desarrollo de la consciencia lingüística de los alumnos; esto último resultó en un estudio cuasi-experimental, de intervención didáctica y análisis estadístico, con el fin de medir la consciencia lingüística de los alumnos en el inicio y al final de la propuesta, y comprobar la eficacia de la intervención.

El estudio contó con la participación de 32 profesores del 1er Ciclo de escuelas públicas de Lisboa y 91 alumnos de edades entre 6 y 7 años. Inicialmente, los educadores frecuentaron un curso de formación, con duración de 50 horas. En el mismo periodo, fue elaborado el instrumento didáctico utilizado como soporte durante la intervención. Es importante añadir que, 
entre los 32 participantes, 5 profesores fueran elegidos, de manera aleatoria, para hacer parte del grupo experimental. Este grupo, a su vez, tuvo una formación complementaria y participó de forma activa en el proceso de reflexión y planificación de la propuesta, además de otras actividades como la selección de producciones hechas por los alumnos, observación de las clases, etc. Así, el experimento fue dividido en cinco fases: formación de profesores y elaboración del trayecto didáctico, prueba previa, implementación del trayecto didáctico, prueba posterior y análisis de datos.

En relación a los trayectos, el PerGRam se centra en tres principales: Triagem, Definição Verbal y Manipulação Frásica. Para cada uno, se desarrolló un conjunto de folletosii, 11 en total, con un breve encuadramiento teórico, objetivos, contenidos y actividades. El folleto 1, intitulado Ponto de Partida, como el propio título sugiere, se trata del inicio de la propuesta y tiene como objetivo principal ayudar el profesor a direccionar los alumnos sobre las actividades que serán realizadas, a través de la identificación de la modalidad de trabajo y del tipo de instrucción.

Los folletos de 2 a 6 fueron elaborados para el trayecto Triagem, y en ellos se presentan las categorizaciones y clasificaciones. En los folletos 2 y 3, Categorização I y Categrização II, la propuesta se centra en los criterios para construcción de conjuntos, etapa apuntada en el material como importante, pues, a partir de la comprensión de que los conceptos van ligados a la categorización, los estudiantes pueden asociar los conceptos a las palabras. Así, las actividades propuestas en los folletos proponen que el profesor divida la clase en grupos y, por medio de imagines y bloques lógicos, los alumnos tienen que discutir posibles categorizaciones y justificar la constitución de los conjuntos formados, que puede ser por tipo de objeto (coches, ropas, etc.), colores, formas, entre otros.

En los folletos 4, 5 y 6 (Triagem de Palavras 1, Triagem de Palavras 2 y Triagem de Palavras 3), en este orden, los objetivos son desarrollar las conciencias de fronteras de palabras, sintáctica y de clases de palabras, teniendo algunos textos como puntos de partida. La primera consciencia está parcialmente estabilizada en los años iniciales de la escuela, excepto por la consciencia de la unidad lingüística palabra, propiamente, que no se encuentra muchas veces fijada en las primeras edades todavía, pudiendo generar equívocos durante la escritura, de ahí la contextualización del material. De esa forma, en los folletos son sugeridas actividades regulares de segmentación, manipulación, reconstrucción de frases y recuento de palabras. La segunda consciencia es un poco más compleja y exige la estimulación de la observación y manipulación de frases, sintagmas y palabras. De este modo, las actividades propuestas para el 
desarrollo de la consciencia sintáctica también engloban ejercicios para la estimulación de la consciencia de la clase de palabras. Como consta en el material, actividades de segmentación y reconstrucción de frases permiten estabilizar la consciencia de las unidades frase y sintagma/grupo, y también la exploración de las regularidades tocantes al orden de las palabras en la lengua portuguesa. Así, teniendo como punto de partida la propuesta de Santos et al. (2014), a partir de la actividad “Tri de Mots” creada por Tisset (2005), las autoras de los folletos defienden que las tareas destinadas a la consciencia sintáctica deben estar apoyadas en textos, que requieren una lectura previa junto a los alumnos, teniendo en cuenta las competencias de lectura, escritura y oralidad. Ese es un paso importante para que el léxico y las estructuras sintácticas presentes en los textos no hagan daño a la eficacia de la actividad.

De esta manera, utilizando conocimientos previos, por medio del manejo de frases y palabras, los estudiantes pueden establecer criterios que posibiliten la organización de las palabras en conjuntos. Las autoras mencionan que este tipo de tarea, realizada conjuntamente, potencializa la actividad metalingüística, entendida como la "concientización explícita de una atención al lenguaje como artefacto, y el monitoreo y manipulación conscientes de la lengua para crear significados deseados basados en entendimientos socialmente compartidos" (Myhill, 2011, p. 250). Esta es una cuestión central en el presente proyecto.

Los folletos de 7 a 9 (Definição Verbal I, Definição Verbal II y Definição Verbal III), como los propios nombres expresan, son destinados al trayecto Definição Verbal y tienen como objetivo principal el desarrollo de la consciencia lexical, a partir de la exploración conceptual y semántica de las palabras, construcción de mapas semánticos, establecimiento de relaciones semánticas entre palabras (relaciones de heteronimia y relaciones parte-todo) y realizaciones de tareas de definición verbal. De acuerdo con Laranjeira, Leite \& Pereira (2015), el desarrollo lexical es muy importante para el establecimiento del lenguaje oral y escrito y por eso necesita ser estimulado en los primeros años de la escuela. Así, entre las vertientes existentes, en los folletos se observa la atención en dos: consciencia del significado de las palabras y consciencia de las relaciones semánticas entre las palabras. La primera asume que tener conocimiento de una palabra sugiere, también, el conocimiento de su significado, y la precisión de éste está relacionada con las experiencias del sujeto. Por eso, las elaboradoras afirman que uno de los procesos que permiten medir de manera más eficiente la precisión del significado de una palabra es la definición verbal. La segunda se refiere a las relaciones asociativas, una vez que desde su exploración, a través del establecimiento de jerarquías, proporciona la construcción y ampliación de redes conceptuales y semánticas que son asociadas a cada palabra. Tareas que 
beneficien la formación de relaciones facilitan el desarrollo de la capacidad de definición verbal de los estudiantes. En este sentido, las dos vertientes elegidas para el material son complementares y ayudan el profesor en el momento de crear sus propios materiales.

Por último, los folletos 10 y 11, intitulados Manipulação Frásica I y Manipulação Frásica II, son destinados al tercero trayecto, Manipulação Frásica, y tienen como objetivo principal desarrollar la consciencia sintáctica y semántica, a través de actividades de manipulación del orden de las palabras en frases y construcción de frases que tengan en cuenta las propiedades semánticas de las palabras. El material persigue que los alumnos sean activos en el proceso, participando y reflexionando como investigadores que deben encontrar las reglas de organización de las frases, más allá de la simple memorización. De este modo, tareas desarrolladas para el establecimiento de las consciencias sintácticas y semánticas pueden favorecer la formación de estructuras más complejas, que serán más exploradas en los Ciclos posteriores, además de ayudar en la consolidación de estructuras aprendidas aún en el 1er Ciclo, por ejemplo.

Consideramos que el proyecto PerGRam constituye una propuesta relevante y original para abordar las necesidades de la enseñanza de la gramática del Portugués como lengua materna, diagnosticadas y presentadas en algunos trabajos centralizados en el contexto de Portugal (Cardoso, Pereira \& Silva, 2015; Ferreira, 2013, entre otros). Además, presenta esfuerzos pertinentes para cambiar las prácticas de la enseñanza de lengua en la escuela, en especial en los primeros años, que pueden extenderse a otros países que tienen el Portugués como lengua materna. Ello es así por cuanto son escasos aún los trabajos que tengan la misma dirección, o sea, que busquen una enseñanza de la gramática reflexiva, teniendo la actividad metalingüística como herramienta importante para el desarrollo de la consciencia lingüística de los estudiantes.

El proyecto apunta y sugiere propuestas innovadoras, más allá de tratamiento habitual de la gramática en el aula, con un sesgo puramente normativo que tiene la lengua estándar como ideal lingüístico a seguir, dando por el contrario espacio a un proceso más activo y reflexivo por parte de profesores y alumnos. Así, los docentes pueden pensar sobre sus prácticas y crear nuevas propuestas, a partir de las sugerencias del proyecto, y los alumnos van a reflexionar sobre la lengua en conjunto, a partir de las actividades propuestas, lo que proporciona un aprendizaje más significativo y duradero.

Así, tres aspectos son interesantes y merecen destaque: la formación de profesores, a través de la presentación de métodos y estudios actuales; la intervención en los años iniciales 
de la escuela, en el 1er Ciclo, asumiendo que desarrollar la consciencia lingüística en el inicio de la formación es beneficioso para desarrollos futuros (Laranjeira, Leite \& Pereira, 2015); y el estimulo de la actividad metalingüística como herramienta para el estabelecimiento de la consciencia lingüística de los estudiantes, aspecto bastante explorado actualmente, pues, como apuntan las investigaciones (Myhill, 2011; Fontich, 2016; Fontich \& García-Folgado, 2018), tareas que generan discusión, reflexión y participación proporcionan mejores desempeños de los alumnos. Finalmente, es interesante apuntar que por tener en cuenta investigaciones de la comunidad científica internacional, la propuesta incentiva la reflexión sobre los nuevos planteamientos para la didáctica de la lengua más allá del contexto portugués, en escenarios cuyo interés sea la enseñanza de la lengua materna.

\section{Referencias}

Cardoso, A., Pereira, S., Pinto, M., \& Silva, E. (2018). O ensino da gramática pela descoberta: o projeto PerGRam. In P. Osório, E. Leurquin \& M. C. Coelho (Ed.), Lugar da gramática na aula de Português (pp. 276-289). Rio de Janeiro: Dialogarts

Ferreira, P. (2013). Ensino da gramática, currículo e terminologia: um estudo multicasos. In M. Teixeira, L Santos, I. Silva, \& E. Mesquita, E. (Ed.), Ensinar e aprender português num mundo plural (pp. 591-628). Lisboa: ESES/UFU.

Fontich, X. (2016). L1 Grammar instruction and writing: Metalinguistic activity as a teaching and research focus'. Language and Linguistics Compass 10 (5), 238-254. DOI: https://doi.org/10.1111/lnc3.12184

Fontich, X.\& García-Folgado, M.-J. (2018). Grammar instruction in the Hispanic area: The case of Spain with attention to empirical studies on metalinguistic activity. L1Educational Studies in Language and Literature 18, 1-39. DOI: https://doi.org/10.17239/L1ESLL-2018.18.04.02

Laranjeira, R., Leite, T., \& Pereira, S. (2015) Desenvolvimento lexical: Perspetivas e práticas de professores no $1 .^{\circ}$ ciclo do ensino básico. Atas do $2^{\circ}$ encontro de mestrados em educação e ensino da ESELx (pp. 173-185). Lisboa: CIED.

Myhill, D. A (2011). The ordeal of deliberate choice: metalinguistic development in secondary writers. In V. Berninger (Ed), Past, present and future contributions of cognitive writing research to cognitive psychology (pp. 247-274). New York: Psychology Press/Taylor Francis Group. 
Santos, A.R., Cardoso, A., \& Pereira, S. (2014). Às voltas com as palavras: desenvolvimento da consciência linguística no $1^{\circ}$ ano de escolaridade. Tejuelo 10, 84-100.

\footnotetext{
${ }^{\text {i }}$ En los trabajos de Cardoso, Pereira \& Santos (2014) y Ferreira (2013), las autoras hacen un panorama interesante sobre la enseñanza de la lengua portuguesa en el aula y, también, sobre la necesidad de unir la enseñanza y la investigación en las prácticas, cuestión recogida en la propuesta del Proyecto PerGRam.

ii Todos los folletos, con contextualización, orientaciones y actividades, están disponibles y pueden ser descargados a través del link: https://www.eselx.ipl.pt/comunidade/recursos/pergram/recursos-online.
}

\section{Informaciones sobre la autora}

Míriam Pessôa Marques, maestra en Letras y Lingüística, graduada en Letras en la Universidad Federal de Alagoas y graduada en Psicología en el Centro Universitario Cesmac. Actualmente es investigadora de doctorado en el Programa de Pós-graduação em Letras e Lingüística (PPGLL/UFAL/Brasil), tutora del Curso de Letras a distancia de UFAL e integrante del grupo de investigación Escritura, Texto e Criação (ET\&C). Tiene experiencia en el área de Lingüística, con énfasis en la enseñanza de lengua y adquisición de la escrita, actuando principalmente en los siguientes temas: escrita colaborativa y generación de ideas en el proceso de escritura.

E-mail: miriam.pess.m@gmail.com

Para citar este artículo:

Pessôa, M. (2019). Projeto PerGRam - Processos para o ensino da gramática no primeiro ano de escolaridade. Bellaterra Journal of Teaching \& Learning Language \& Literature 12(2), 102-107. DOI: https://doi.org/10.5565/rev/jt13.814 